\title{
ACYCLIC SEMIGROUPS AND MULTIPLICATIONS ON TWO-MANIFOLDS
}

\author{
BY \\ HASKELL COHEN AND R. J. KOCH(1) \\ TO PROFESSOR A. D. WALLACE ON HIS SIXTIETH BIRTHDAY
}

1. Introduction. We are concerned here with some aspects of acyclicity in a compact connected Hausdorff topological semigroup $S$, and applications to the admissibility of certain multiplications on two-manifolds and related spaces. Our main result is that if $S$ is a semigroup on a compact connected two-manifold without boundary, then $S=E S E \Rightarrow S=K$ (see definitions below). This is a partial solution to a problem proposed by A. D. Wallace, namely, if $S$ is a two-sphere, does $S=S S$ imply $S=K$ ? Our result holds also for spaces obtained from two-manifolds by successively shrinking retracts to points. It follows that if $S=E S E$, the multiplications on $S$ must be either left trivial $(x y=x$, all $x, y)$ or right trivial $(x y=y$, all $x, y$ ) except in the case of the torus $=C \times C$ where the multiplication in either factor may be that of a group, or left trivial, or right trivial.

We also give some generalizations of results of Wallace [10] dealing with acyclicity of certain compact connected semigroups. In particular we show that if $S$ is compact and connected with zero, if $S=E S$ and if either $S$ is regular (i.e. $a \in S \Rightarrow a \in a S a$ ) or the idempotents commute, then $S$ is acyclic.

The notation is that of [7]. In particular $K$ denotes the minimal ideal, $E$ is the set of idempotents. For a closed subset $A$ of $S, S / A$ is the space obtained by shrinking $A$ to a point. The cohomology used is that of Alexander-Wallace-Spanier with coefficient group arbitrary unless otherwise specified. Throughout the paper we shall use reduced groups in dimension 0 . The symbol $\approx$ denotes isomorphism onto, $\sim$ denotes isomorphism into. We denote by $A \backslash B$ the complement of $B$ in $A$, the closure of $A$ by $A^{*}$, and the empty set by $\square$.

2. Results on acyclicity. We first state without proof a theorem due to Wallace [9] which will be of importance in the sequel.

THEOREM 1. Let $X$ and $Y$ be compact Hausdorff spaces, and let $R$ be a closed subset of $X \times Y$. For $B \subset Y$, let $L(B)=\operatorname{proj}_{1}[(X \times B) \cap R]$. If

(i) $L(A) \cap L(B)$ is connected for each pair of closed subsets $A$ and $B$ of $Y$ and

(ii) $H^{1} L(y)=0$, each $y \in Y$, then $H^{1} L(A)=0$, each closed subset $A \subset Y$. If also

Received by the editors August 5, 1963.

(1) This research was supported in part by the National Science Foundation under Grant NSF-G14085. 
(iii) Whenever $A$ and $B$ are closed subsets of $Y, L(A) \cap L(B)=L(C)$ for some closed subset $C \subset Y$ and

(iv) $H^{p} L(y)=0$ for each $y \in Y, 1 \leqq p \leqq q$,

then $H^{p} L(A)=0$ for each closed subset $A \subset Y, 1 \leqq p \leqq q$.

Note, in particular, that if $X \subset Y$ and $R$ is reflexive and transitive, then $L(A) \cap L(B)=L(L(A) \cap L(B))$ for subsets $A$ and $B$ of $Y$. So, in this case, (iii) holds.

LEMMA 1. Let $S$ be a compact connected semigroup with zero (0), let $L$ be a closed left ideal in $S$, and $e^{2}=e \in S$. Then $H^{p}(e L)=0, p=0,1,2, \cdots$.

Proof. Note that $S$ acts on $e L: t(e x)=e t e x$. Hence we have the diagrams

$$
\frac{e L \stackrel{t}{\rightarrow} e t e L \stackrel{i}{\subset} e L}{f_{t}}, \frac{H^{p}(e L) \stackrel{i^{*}}{\rightarrow} H^{p}(e t e L) \stackrel{t^{*}}{\rightarrow} H^{p}(e L)}{f_{t}^{*}},
$$

where $i$ denotes inclusion and $f_{t}$ is defined by composition. By the homotopy theorem [6], $0=f_{0}^{*}=f_{e}^{*}=i d$, so $H^{p}(e L)=0$.

THEOREM 2. Let $S$ be a compact connected semigroup with zero, $S=E S E$, and $I$ a closed ideal of $S$. Then $H^{1}(A I)=0$ for each closed subset $A$ of $E$; in particular $H^{1}(I)=0$.

Proof. Define $R \subset S \times E$ by $(t, e) \in R \Leftrightarrow t \in e I$. Then $L(A) \cap L(B)=A I \cap B I$ is a right ideal in $S=S E$ and is therefore connected for each $A, B$ closed. By Lemma $1, H^{1}(e I)=0$, and the conclusion follows from Theorem 1 .

There are unpublished $\left({ }^{2}\right)$ examples of A.L. Hudson to show that "ideal" cannot be replaced by "right ideal", and that $H^{2}(S)$ may be nonzero. Also if only $S=E S$ is assumed, $H^{1}(S)$ may be nonzero.

Corollary 1. Let $S$ be a compact connected semigroup with $S=E S E$, and let $I$ be a closed ideal in $S$; then $H^{1}(I) \approx H^{1}(K)$.

Proof. Let $\tilde{E}$ denote the set of idempotents in $S / K$, the Rees quotient. Let $\phi: S \rightarrow S / K$ be the natural homomorphism. Then $\phi(E)=\tilde{E}$ and it follows that $\phi(S)=\phi(E) \phi(S) \phi(E)$, and $\phi(S)$ has a zero. Now $I / K=\phi(I)$ is an ideal ir $\phi(S)$, so $\phi(E) \phi(I)=\phi(E I)=\phi(I)=I / K$ and $H^{1}(I / K)=0$ by the theorem. Hence $H^{1}(I, K) \approx H^{1}(I / K)=0$.

Consider the exact sequence $H^{1}(I, K) \rightarrow H^{1}(I) \stackrel{i^{*}}{\rightarrow} H^{1}(K)$. Since $H^{1}(I, K)=0, i^{*} \sim$; but $K$ is a retract of $S[8]$, so $i^{*}$ is onto and the proof is complete.

3. Topological lemmas. Let $X$ be a space, and $h \in H^{n}(X)$. If $A \subset X$, then $h \mid A$ denotes the image of $h$ under the natural homomorphism $H^{n}(X) \stackrel{\iota^{*}}{\rightarrow} H^{n}(A)$.

(2) Added in proof. A. L. Hudson, Example of a nonacyclic continuum semigroup $S$ with zero and $S=E S E$, Proc. Amer. Math. Soc. 14 (1963), 648-653. 
We say that $k \in H^{n}(A)$ is extendable to $H^{n}(X)$ (or to $X$ ) if $k$ is in the image of $i^{*}$. If $X$ is compact and $h \in H^{n}(X)$ is not zero, then there exists a closed set $F$ minimal relative to $h \mid F \neq 0 ; F$ is called a floor for $h$.

LeMmA 2. Let $X$ be a compact Hausdorff space such that $H^{n}(X) \neq 0, X$ is a floor for each nonzero $h \in H^{n}(X)$, and let $R$ be a proper closed subset of $X$ satisfying $H^{n-1}(X) \rightarrow H^{n-1}(R)$ is onto. Then $H^{n}(X / R) \neq 0$, and $X / R$ is a floor for each nonzero $h \in H^{n}(X / R)$. In particular, the conclusions hold if $R$ is a retract of $X$.

Proof. Let $\phi: X \rightarrow X / R$ be the natural map; consider the exact sequence $\rightarrow H^{n}(X, R) \rightarrow H^{n}(X) \rightarrow i^{i^{*}} H^{n}(R) \rightarrow$. If $H^{n}(X, R)=0$, then $i^{*} \sim$; but $i^{*}$ is the 0-mapping by the floor properties. Hence $H^{n}(X, R) \neq 0$; let $h$ be a nonzero element of $H^{n}(X / R)$ and let $B$ be a closed proper subset of $X / R$ with $h \mid B \neq 0$. We have the diagram

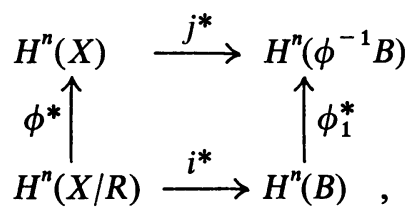

where $j^{*}$ is induced by inclusion, and $\phi_{1}^{*}$ is induced by the restriction of $\phi$ to $\phi^{-1} B$. Since $\phi^{-1} B$ is a proper closed subset of $X$, we conclude from the floor property that $j^{*}$ is the 0 -mapping, so $0=j^{*} \phi^{*}=\phi_{1}^{*} i^{*}$. We will arrive at a contradiction by showing that $\phi_{1}^{*} \sim$, for then $\phi_{1}^{*} i^{*} h \neq 0$. We consider two cases; (1) $0=\phi(R) \notin B$, and (2) $0=\phi(R) \in B$. In case (1), note that $\phi_{1}$ is a homeomorphism onto, so $\phi_{1}^{*} \approx$. In case (2) consider the commutative diagram

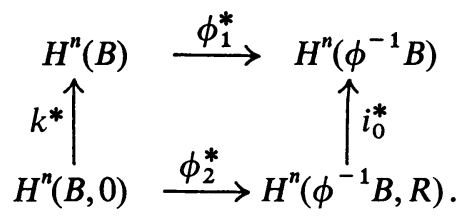

Now $k^{*} \approx, \phi_{2}^{*} \approx$ by the map excision theorem [5]. We claim $i_{0}^{*} \sim$; consider the exact sequence:

$$
\begin{gathered}
H^{n-1}\left(\phi^{-1} B\right) \stackrel{m^{*}}{\longrightarrow} H^{n-1}(R) \\
H^{n}\left(\phi^{-1} B, R\right) \stackrel{i^{*}}{\longrightarrow} H^{n}\left(\phi^{-1} B\right) .
\end{gathered}
$$

Since $H^{n-1}(X) \rightarrow H^{n-1}(R)$ is onto, $m^{*}$ is onto and it follows that $i_{0}^{*} \sim$. Hence $\phi_{1}^{*} \sim$, and the proof is complete.

We note that if $X$ is connected and satisfies the conditions of Lemma 2 and if $H^{n-1}(X) \rightarrow H^{n-1}(R)$ is onto, then $R$ does not separate $X$. We omit the proof, 
which depends on an exact sequence argument using Lemma 2. In particular, a retract or an acyclic continuum of an $n$-manifold $X$ does not separate $X$.

In what follows we use the codimension function of [2]. Recall that for a compact Hausdorff space $X$, cd $X \leqq n$ iff $H^{n}(X) \rightarrow H^{n}(A)$ is onto for each closed subset $A$ of $X$. Also [2], cd $X \leqq n$ iff $H^{n+1}(X, A)=0$ for each closed subset $A$.

Lemma 3. Let $A$ be a closed subset of a compact Hausdorff space $X$. If cd $X \leqq n$, then $\operatorname{cd} X / A \leqq n$.

Proof. Let $\phi: X \rightarrow X / A$ be the natural map, and let $B$ be a closed subset of $X / A$. The proof involves two cases.

Case 1. $0=\phi(A) \in B$ (i.e., $\left.A \subset \phi^{-1} B\right)$. Then $\left(X, \phi^{-1}(B) \cup A\right) \rightarrow^{\phi_{1}}(X / A, B)$, where $\phi_{1}$ is the mapping determined by $\phi$. Note that $\phi_{1}$ is a closed map, and $\phi_{1}\left(X \backslash \phi^{-1}(B) \cup A\right)$ is homeomorphic to $(X / A) \backslash B$. Hence by the map excision theorem $\phi_{1}^{*} \approx$. Now $H^{n+1}\left(X, \phi^{-1}(B) \cup A\right)=0$, so $H^{n+1}(X / A, B)=0$. Hence $\operatorname{cd}(X / A) \leqq n$, and the proof is complete.

Case 2. $0=\phi(A) \notin B$ (i.e., $\phi^{-1} B \cap A=\square$ ). Consider the diagram:

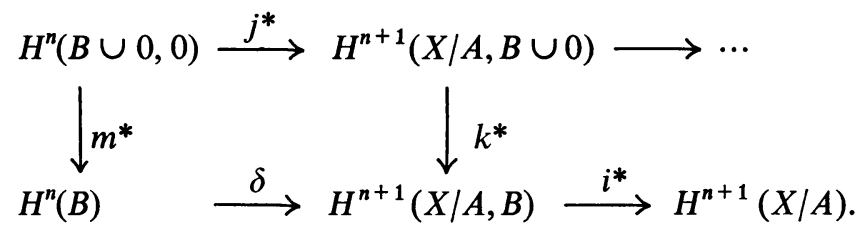

Note that $H^{n+1}(X \mid A, B \cup 0) \approx H^{n+1}\left(X, \phi^{-1} B \cup A\right)=0 ; H^{n+1}(X / A) \approx H^{n+1}(X, A)=0$. Let $h \in H^{n+1}(X / A, B)$; then $i^{*} h=0$, so there exists $h_{1} \in H^{n}(B)$ with $: \delta h_{1}=h$. Note that $m^{*} \approx$; let $h_{2}=m^{*-1}\left(h_{1}\right)$. Then $h=\delta m^{*}\left(h_{2}\right)=k^{*}\left(j^{*} h_{2}\right)=k^{*}(0)=0$. Thus $H^{n+1}(X / A, B)=0$ and the proof is complete.

\section{Some semigroups on manifolds and related spaces.}

THEOREM 3. Let $S$ be a compact connected semigroup satisfying (i) $S=E S E$, (ii) $H^{2}(S) \neq 0$, (iii) $S$ is a floor for each nonzero $h \in H^{2}(S)$, (iv) cd $S \leqq 2$; then there exists $e \in E$ such that $S=S e S$.

Proof. Suppose first that $S$ has a zero (0). Then $H^{1}(S a S)=0$ for each $a \in S$ by Theorem 2. Define $a \leqq b$ iff $S a S \subset S b S$. Then $\leqq$ has closed graph, and is reflexive and transitive; hence (iii) of Theorem 1 holds. Note that for $A, B \subset S$ $L(A) \cap L(B)$ is an ideal of $S=E S E$; hence $L(A) \cap L(B)$ is connected, so (i) of Theorem 1 holds. Since $L(a)=S a S$ and $H^{1}(S a S)=0$, (ii) of Theorem 1 holds. If $H^{2}(S a S)=0$ for each $a$, then by Theorem $1, H^{2}(S)=0$. Therefore there exists $a \in S$ such that $H^{2}(S a S) \neq 0$. Let $h$ be a nonzero element of $H^{2}(S a S)$. Since cd $S \leqq 2, h$ can be extended to $h_{1} \in H^{2}(S)$. If $S a S<S$, then since $S$ is a floor for $h_{1}$, we conclude that $h=0$, a contradiction. Therefore $S a S=S$ and since $S=E S E$, there exists $e \in E$ with $a=e a$. Hence $S=S a S=S e a S \subset S e S$ and so $S=S e S$. 
If $S$ has no zero, consider $\phi: S \rightarrow S / K$, the Rees quotient. If $S=K$, the conclusion is immediate, so we assume $K<S$. Then $S / K$ has a zero, and satisfies (i). By Lemma 2, $S / K$ satisfies (ii); and by Lemmas 2 and 3, $S / K$ satisfies (iii) and (iv). Hence by the first part of the proof, $S / K=S / K \phi(e) S / K$ for some $e \in K$. It follows that $S=S e S$, and the proof is complete.

LEMMA 4. Let $S$ be a compact connected semigroup with left unit e. If e has a neighborhood $U$ homeomorphic to Euclidean $n$-space $R^{n}$, then e belongs to $K$, and $S$ is a minimal right ideal.

Proof. Let $V$ be an open set homeomorphic to $R^{n}$, with $e \in V \subset V^{2} \subset U$. Identify $U$ with $R^{n}$, metric $d$. Fix $\varepsilon>0$ such that $N_{\varepsilon}(e) \subset V$ and let $Q=N_{\varepsilon}(e)^{*}$. Since the multiplication is uniformly continuous on $Q$, there exists $\delta>0$ such that $y \in N_{\delta}(e) \rightarrow d(x, y x)<\varepsilon$ for each $x \in Q$. Hence for each $y \in N_{\delta}(e)$, left translation by $y$ takes $Q$ into $R^{n}$. Using the Brouwer fixed point theorem (see [4, Lemma 1.1]) there exists $q \in Q$ with $y q=e$. Hence each $y \in N_{\delta}(e)$ has a right inverse, or alternatively $R_{e}=\{y: e \in y S\}$ has an interior. Now suppose $e \notin K$ and we will show that $R_{e}$ is nowhere dense, a contradiction. Let $I_{R}=\{y \in S: e \notin y S\}$. Then $I_{R}$ is easily seen to be an open right ideal [3]. By the connectedness, $I_{R}^{*} \neq I_{R}$, so $R_{e} \cap I_{R}^{*} \neq \square$. It follows that $R_{e} \subset I_{R}^{*}$. Hence $I_{R}$ is open and dense, and the proof is complete.

The initial part of the proof of Lemma 4 follows closely the argument of a theorem of Mostert and Shields [4] which states: If the unit of a semigroup has a Euclidean neighborhood, then the maximal group containing the unit is open. As a consequence if $S$ is a compact connected manifold which is a semigroup with unit, then $S$ is a group. (See also [6].) If only a left unit is assumed, then by Lemma 4 we conclude that $S=K$, so $S$ is the union of groups.

THEOREM 4. Let $S$ be a compact connected semigroup satisfying

(i) $S=E S E$;

(ii) $H^{2}(S) \neq 0$;

(iii) $S$ is a floor for each nonzero $h \in H^{2}(S)$;

(iv) $c d S \leqq 2$; and

(v) $S$ is locally Euclidean except possibly at one point; then $S=K$.

Proof. Suppose $S \neq K$. Then $S / K$ is compact, connected with zero and satisfies (i), (ii), (iii), (iv) and may be non-Euclidean at perhaps 2 points, one of which is zero. Hence we may keep the original notation and assume $S$ has a zero. It follows from Theorem 2 that $H^{1}(S)=0$. We show first that $e S \cup f S \neq S$ for any $e, f$ in $E$. Suppose $S=e S \cup f S$. If $e$ is zero, then $S=f S$; but $f S$ is compact, connected, has left unit and zero, and is hence acyclic, a contradiction. Hence $e \neq 0 \neq f$, so one of $e, f$ has small Euclidean neighborhoods, say $e$. Now $e \notin f S$, so there is a Euclidean neighborhood $U$ such that $e \in U \subset S \backslash f S$. Then $e \in U \subset e S$, so by Lemma $4, e=0$, a contradiction. We show next that for idempotents $e$ and $f$. $H^{1}(e S \cap f S)=0$. Consider the Mayer-Vietoris sequence 
1965] ACYCLIC SEMIGROUPS AND MULTIPLICATIONS ON TWO-MANIFOLDS 425

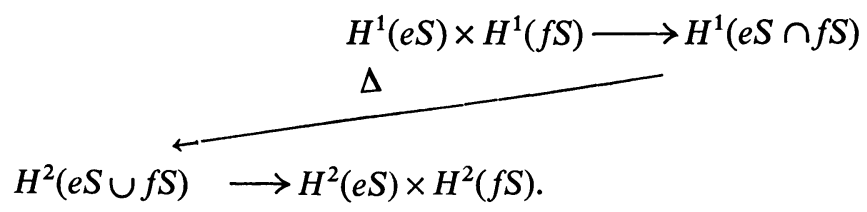

Note that $H^{i}(e S), H^{i}(f S)$ are 0 for $i=1,2$, hence $\Delta \approx$. If $0 \neq h \in H^{2}(e S \cup f S)$, then since $\operatorname{cd} S \leqq 2$, there is $h_{1} \in H^{2}(S)$ with $h_{1} \mid e S \cup f S=h$. But $S$ is a floor for $h_{1}$, hence $h=0$. Hence $H^{2}(e S \cup f S)=0=H^{1}(e S \cap f S)$.

Define $R \subset S \times(E \times E)$ by $(x, e, f) \in R$ iff $x \in e S \cap f S$. Note that $R$ is closed, and for $(e, f) \in E \times E, L(e, f)=e S \cap f S$ is a right ideal in $S=S E$ with zero and is therefore connected. If $C$ is a closed subset of $E \times E$ it follows that $L(C)$ is connected. Since $H^{1} L(e, f)=0$ we conclude by Wallace's Theorem that $H^{1} L(C)=0$ for each closed subset of $E \times E$. In particular, if $A$ and $B$ are closed subsets of $E$, then $L(A \times B)=A S \cap B S$, and $H^{1}(A S \cap B S)=0$.

Now let $h$ be a nonzero element of $H^{2}(S)$, and let $F$ be a subset of $E$ which is minimal relative to being closed and satisfying $h \mid F S \neq 0$. (We are using here the hypothesis that $S=E S$.) If $F$ is a single element $e$, then since $e S$ is acyclic we have a contradiction. Hence $F=A \cup B$, where $A$ and $B$ are closed, $A \notin B, B \notin A$. Consider the exact sequence

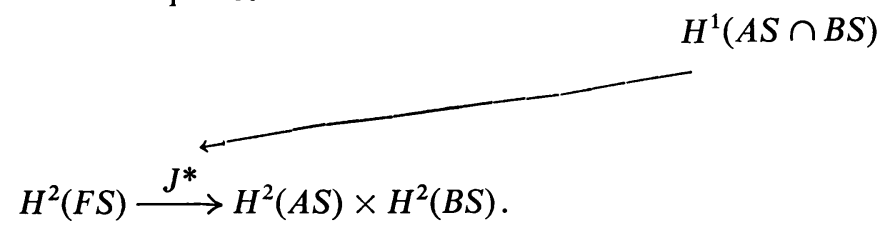

Since $H^{1}(A S \cap B S)=0, J^{*}$ is an isomorphism into. Let $h \mid F S=h_{0}$. Then $h_{0}\left|A S=0=h_{0}\right| B S$ by the minimality of $F$, hence $J^{*}\left(h_{0}\right)=0$. Therefore $h_{0}=0$, a contradition which completes the proof.

Lemma 5. Let $X$ be a metric continuum satisfying (i) $H^{1}(S)=0$, (ii) $H^{2}(S) \neq 0$, (iii) $S$ is a floor for each nonzero $h \in H^{2}(S)$, (iv) $\operatorname{cd} S \leqq 2$, and ( $\left.\mathrm{v}^{\prime}\right)$ $S$ is locally Euclidean except possibly at a finite number of points; then $S$ is homeomorphic to the 2-sphere. (The coefficient group here is taken to be the integers modulo a prime.)

This follows from the fact that $S$ is locally connected, and by using Zippin's characterizations of the two-sphere. We omit the proof. We note that the restriction on the coefficient group rules out the projective plane.

COROLlaRY 2. Let $S$ be a semigroup which is a metric continuum satisfying (i)-(iv) and $\left(\mathrm{v}^{\prime}\right)$; then $S=K$.

Proof. If $K<S$, then $S / K$ is a semigroup with zero satisfying the conditions of Lemma 5, as follows: (i) follows from Theorem 2, (ii) follows from Lemma 2, (iii) and (iv) follow from Lemmas 2 and 3 , and $\left(v^{\prime}\right)$ is clear. Hence $S / K$ is a two- 
sphere, and therefore satisfies (i)-(v) of Theorem 4 . Hence $S / K=0$, a contradiction, so $S=K$.

REMARKS. The compact connected 2-manifolds without boundary are covered by Theorem 4. An example of the type of space covered by Corollary 2 is a double torus with each of two nonbounding circles pinched to points. Other examples are readily constructed.

As shown in Corollary 2, the metrizability can be used to relax condition (v) of Theorem 4. It would be of interest to know if $S=e S \cup f S$ is possible where both $e$ and $f$ are non-Euclidean points. A negative answer would render the metrizability unnecessary in Corollary 2.

\section{Further results on acyclicity.}

Definitions. Let $S$ be a compact semigroup, $a \in S$. Define $T_{a}=\{x: x a=a\}$; let $E_{a}=E \cap T_{a}$, and let $K_{a}$ be the kernel of $T_{a}$. Define $\leqq$ on $S \times S$ by: $a \leqq b$ iff $E_{b} \subset E_{a}$. Let $L(a)=\{x: x \leqq a\}$. Recall that a semigroup is right simple if it is a minimal right ideal of itself.

LEMMA 6. Let $S$ be a compact semigroup; then $\leqq$ has closed graph and is reflexive and transitive. Further, for $a \in E$,

$$
L(a)=\bigcap\left\{f S: f \in E_{a}\right\}=\bigcap\left\{f S: f \in E_{a} \cap K_{a}\right\} .
$$

If also $K_{a}$ is right simple, then $L(a)=f S$ for each $f \in E \cap K_{a}$, and is acyclic if $S$ is connected and has a zero.

Proof. It is easy to see that $\leqq$ has closed graph, and is reflexive and transitive. Let $x \in L(a)$ and $f \in E_{a}$; then $f \in E_{a} \subset E_{x}$, so $f x=x$ and $x \in f S$. Hence $L(a) \subset \bigcap\left\{f S: f \in E_{a}\right\}$, and the latter is clearly contained in $\bigcap\left\{f S: f \in E_{a} \cap K_{a}\right\}$. Let $z \in \bigcap\left\{f S: f \in E_{a} \cap K_{a}\right\}$, and let $e \in E_{a}$. Then $e T_{a}$ contains a minimal right ideal $e_{0} T_{a}$ of $T_{a}$, where $e_{0} \in E_{a} \cap K_{a}$. Hence $z \in e_{0} S \subset e S$, so $e z=z$, and $e \in E_{z}$. Therefore $\bigcap\left\{f S: f \in E_{a} \cap K_{a}\right\} \subset L(a)$. The remaining statements are clear, the acyclicity of $L(a)$ following from [6].

REMARK. By analogous arguments it can be seen that if $S$ is compact and connected, and if $K_{t}$ is right simple for each $t \in S$, then the relation $\leqq_{1}$ on $S a \times S$ defined by $x \leqq_{1} y$ iff $x \in e S a$ where $e \in E_{y}$ and $a$ is fixed, is reflexive and transitive. Further $L(y)=e S a$ is acyclic by Lemma 1 . Note that $\leqq_{1}$ is the restriction of $\leqq$ to $S a \times S$.

LEMMA 7. Let $S$ be a compact connected semigroup with zero (0), with $S=E S$ and $K_{a}$ right simple for each $a \in S$; then $e S \cap f S$ is connected for each $e, f \in E$. Hence if $A$ and $B$ are subsets of $E$, then $A S \cap B S$ is connected.

Proof. Let $x \in e S \cap f S$; then $e, f \in T_{x}$. Let $g^{2}=g \in K_{x}$; since $K_{x}$ is right simple, $K_{x}=g K_{x}=g T_{x}$. Hence $g T_{x} \subset e T_{x} \cap f T_{x}$, so $g \in e S \cap f S$. Therefore $x \in g S \subset e S \cap f S$. Hence 0 and $x$ lie in the connected subset $g S$ of $e S \cap f S$, and the conclusion follows. 
REMARK. An analogous argument shows that $A S a \cap B S a$ is connected, for each $A, B \subset E, a \in S$.

Notes. For a semigroup $S$ we see that $S=E S$ implies $T_{a} \neq \square$ for each $a$. If further $S$ is compact, then $\square \neq K_{a}$ is compact and simple, hence completely simple. If the idempotents commute, it follows that $K_{a}$ is a group, and in particular right simple. If $S$ is regular, then (even without compactness) $\{x: S x a=S a$ and $x a S=a S\}$ has right simple kernel $\bigcup\left\{H_{e}: e S=a S\right\}[1$, p. 66], i.e., those group elements which generate the same right ideal which $a$ generates. It follows that $K_{a}$ exists and coincides with $\{f \in E: f S=a S\}$, and is right simple.

THEOREM 5. Let $S$ be a compact connected semigroup with $S=E S$ and $K_{a}$ right simple for each $a \in S$; then $H^{n}(S) \approx H^{n}(K)$ for all $n$.

Proof. Define $\leqq$ on $S \times S$ by $x \leqq y$ iff $x \in e S$ where $e \in E_{y}$. By Lemmas 6 and 7 , the hypotheses of Theorem 1 are fulfilled, so $H^{\prime \prime}(S)=0$ for each $n$. If $K$ is nondegenerate, consider the Rees quotient $S / K$. Note that $S / K$ satisfies the conditions on $S$, and has a zero, hence $H^{n}(S / K)=0$ for each $n$. From the exact sequence $H^{n}(S, K) \rightarrow H^{n}(S) \rightarrow H^{n}(K)$ we conclude that $H^{n}(S) \approx H^{n}(K)$ for all $n$.

REMARK. By using the remarks following Lemmas 6 and 7, a similar argument will establish the stronger conclusion $H^{n}(S) \approx H^{n}(L)$ for each closed left ideal containing $K$. For the sake of clarity of exposition we have chosen to give the proof of the less general result. In particular we have

COROLlary 3. If $S$ is a compact connected semigroup with zero and $S=E S$ and if either (1) $S$ is regular or (2) $E$ is commutative, then each closed left ideal is acyclic.

We note that this extends some results of Wallace [10].

\section{REFERENCES}

1. A. H. Clifford and G. B. Preston, The algebraic theory of semigroups, Math. Surveys No. 7, Amer. Math. Soc., Providence, R.I., 1961.

2. Haskell Cohen, A cohomological definition of dimension for locally compact Hausdorff spaces, Duke Math. J. 21 (1954), 209-224.

3. R. J. Koch and A. D. Wallace, Maximal ideals in compact semigroups, Duke Math. J. 21 (1954), 681-686.

4. P. S. Mostert and A. L. Shields, Semigroups with identity on a manifold, Trans. Amer. Math. Soc. 91 (1959), 380-389.

5. A. D. Wallace, The map excision theorem, Duke Math. J. 19 (1952), 177-182.

6. - Cohomology, dimension and mobs, Summa Brasil. Math. 3 (1955), 45-54.

7. - The structure of topological semigroups. Bull. Amer. Math. Soc. 61 (1955), 95-112.

8. —, Retractions in semigroups, Pacific J. Math. 7 (1957), 1513-1517.

9. —_, A theorem on acyclicity, Bull. Amer. Math. Soc., 67 (1961), 123-124.

10. — Acyclicity of compact connected semigroups, Fund. Math. 50 (1961), 99-105.

Louisiana State University,

BATON ROUGE, LouisianA 